\title{
Regge spectra, symmetry-breaking effects, and decays of old and new mesons in dual resonance amplitudes
}

\author{
Masaaki Kuroda* \\ Deutsches Elektronen-Synchrotron DESY, Hamburg, Germany \\ Bing-Lin Young ${ }^{\dagger}$ \\ Deutsches Elektronen-Synchrotron DESY, Hamburg, Germany \\ and Ames Laboratory-ERDA and Department of Physics, Iowa State University, Ames, Iowa $50011^{\ddagger}$
}

(Received 14 February 1977)

\begin{abstract}
Single-term Veneziano dual amplitudes with nondegenerate Regge slopes are examined in detail. Factorization of parent resonance residues and the equal-spacing rule extracted from the universal-slope case are used to determine all the leading-meson-trajectory parameters in terms of that of the $\rho$. Relations between nondegenerate slopes and SU(4)-symmetry-breaking effects are discussed. The predicted meson mass spectra and the partial decay widths into two pseudoscalars are shown to agree well with data for the cases in which data exist.
\end{abstract}

\section{INTRODUCTION}

Investigations of the properties of charmed mesons $^{1}$ in the duality scheme have been carried out by several authors. One framework used is the concise dual Veneziano model ${ }^{2}$ which has been successful in dealing with ordinary particles. In order to accommodate the new particles, a modification of the Veneziano model is needed, and this modification takes mainly two forms: (a) incorporating the SU(4) structure in the original Veneziano model with a universal Regge slope ${ }^{3}$ and (b) modifying the original framework to allow different slopes for different meson trajectories. ${ }^{4}$

Scheme (a) is supported by the mass-quantization relation ${ }^{5} \alpha_{D^{*}}\left(m_{D}{ }^{2}\right)=\frac{1}{2}$ (Ref. 3 ) satisfied by the $D$ trajectory with the universal slope $\alpha_{D *}^{\prime}=\alpha_{\rho}^{\prime}$ $=\frac{1}{2}\left(m_{\rho}^{2}-m_{\pi}^{2}\right)^{-1}$. This approach leads to the generalization of the nonet mass formula to SU(4) including vector and pseudoscalar mesons, but it has difficulty in accommodating the $J / \psi$ particle. There is evidence that the $J / \psi$ trajectory possesses a smaller slope. If we take $\psi^{\prime}(3.7)$ as its first daughter, then the slope is $\alpha_{J}^{\prime} \simeq 0.25$, while taking $\psi^{\prime}(3.7)$ as the second daughter gives $\alpha_{J}^{\prime} \simeq 0.5$. Another estimate obtained by taking $\chi(3550)$ as the $2^{++}$exchange degenerate partner of $J / \psi$ gives $\alpha_{J}^{\prime}$ $\simeq 0.33$. The last value of $\alpha_{J}^{\prime}$ leads to $m_{\eta_{c}}=2.84$ $\mathrm{GeV}$, if the Ademollo-Veneziano-Weinberg massquantization rule ${ }^{5} \alpha_{J}\left(m_{n_{c}}{ }^{2}\right)=\frac{1}{2}$ is used, ${ }^{6}$ where $\eta_{c}$ is the $c \bar{c} 0^{-+}$state. In all these cases the slope of the $J / \psi$ trajectory is much smaller than that of the $\rho$ trajectory, $\alpha_{\rho}^{\prime} \simeq 0.9 \mathrm{GeV}^{-2}$.

Scheme (b) is motivated by the drastically different mass scales of the charmed and ordinary mesons which are expected to give rise to appreciable higher-order symmetry-breaking effects.
One of the possible consequences is that coupling constants may significantly deviate from their [SU(4)] symmetry values. This in turn will require that the universality of the trajectory slope parameters be broken in Veneziano amplitudes, as slopes and coupling constants are related there. The presence of higher-order symmetry-breaking effects in the charmed SU(3) sector of SU(4) can be seen from the poorly satisfied charmed nonet mass formula, e.g. $m_{J}{ }^{2}+m_{\rho}{ }^{2}=2 m_{D} *^{2}$, which is good only to $20 \%$.

The Veneziano formula with nondegenerate slopes has a serious problem. It leads to an exponentially increasing scattering amplitude for large fixed angle at high energy. ${ }^{7} \mathrm{~A}$ way out of this difficulty was proposed recently by $\operatorname{Igi},{ }^{8}$ who suggested that the phenomenon of nondegenerate slopes occurs at low energy and that at high energy, where the Regge asymptotic expansion is applied, all the slopes become universal. Although such a proposal is very difficult to implement analytically, it provides an attractive phenomenological framework tailored to the resonance region and may be useful in the description of certain aspects of the new particles.

Many interesting relations have been derived in terms of single-term Veneziano amplitudes in the case of degenerate slopes, such as the nonet mass relations involving vector (tensor) and pseudoscalar mesons, ${ }^{2}$ the equal-spacing rule ${ }^{9}$ the massquantization rule ${ }^{5}$ factorization of the parents and the first daughters, ${ }^{10}$ etc. (They are not all independent.) Further, the couplings of two pseudoscalar mesons to all the particles on a leading trajectory are related to each other, and these couplings of the particles of the same spin, but lying on different trajectories, satisfy SU(3) symmetry. 
Undoubtedly, some of these results will no longer be true in the case of nondegenerate slopes.

In this article, restricting ourselves essentially to the resonance region, we study in detail the properties of the Veneziano model with nondegenerate slopes. We examine the factorization property and propose a scheme to express all the slopes of the leading meson trajectories in terms of, say, that of the $\rho$ trajectory, and thereby determine them unambiguously. ${ }^{11}$

In Sec. II we review briefly some of the results known in the case of universal slopes. In particular, we put the factorization conditions in a form such that we can adopt them to the case of nondegenerate slopes. Section III treats the nondegenerate slopes. In Sec. IV, we present a discussion of the symmetry-breaking effects and calculate the meson mass spectra and the partial widths of the leading $1^{-}, 2^{+}, 3^{-}$mesons decaying into two pseudoscalar mesons. We also predict the mass of the $F^{*}$. Our conclusions are given in Sec. $\mathrm{V}$.

\section{FACTORIZATION}

To put things in perspective, let us recaptiulate some of the known results involving only the old mesons. Consider the following $s$-channel processes with their single-term Veneziano amplitudes:

$$
\begin{aligned}
\boldsymbol{A}\left(\pi^{+} \pi^{-}-\pi^{+} \pi^{-}\right) & =\lambda^{\pi} \boldsymbol{V}_{\rho \rho}(s, t), \\
A\left(\pi^{+} \pi^{-} \rightarrow K^{+} K^{-}\right) & =\lambda^{\pi K} V_{\rho K^{*}}(s, t), \\
\boldsymbol{A}\left(K^{+} K^{-}-K^{+} K^{-}\right)=\frac{1}{2} \lambda^{K} & {\left[V_{\rho \phi}(s, t)+V_{\phi \rho}(s, t)\right.} \\
& \left.\quad V_{\omega \phi}(s, t)+V_{\phi \omega}(s, t)\right]
\end{aligned}
$$

where

$$
V_{a b}(s, t) \equiv \frac{\Gamma\left(1-\alpha_{a}(s)\right) \Gamma\left(1-\alpha_{b}(t)\right)}{\Gamma\left(1-\alpha_{a}(s)-\alpha_{b}(t)\right)},
$$

and $\alpha_{\rho}, \alpha_{\omega}, \alpha_{K^{*}}$, and $\alpha_{\phi}$ represent the exchangedegenerate $\rho-f-\omega-A_{2}, K^{*}-K^{* *}$, and $\phi-f^{\prime}$ trajectories.

We first fix the normalization constants $\lambda^{\pi}, \lambda^{\pi K}$, and $\lambda^{K}$ by considering Regge expansions in the $t$ channel for large $t$ and small $s$. Following Igi, ${ }^{8}$ we assume that when $t \rightarrow \infty$, the slopes of the trajectories contributing to the $t$ channel reduce to a universal value. The $\rho$ trajectory exchanged in the $s$ channel leads to

$$
\begin{aligned}
& \lambda^{\pi} \Gamma\left(1-\alpha_{\rho}(s)\right)\left(-\alpha_{\rho}^{\prime} t\right)^{\alpha_{\rho}(s)}\left(1+O\left(\frac{1}{t}\right)\right), \\
& \lambda^{\pi K} \Gamma\left(1-\alpha_{\rho}(s)\right)\left(-\alpha_{K^{*}}^{\prime} t\right)^{\alpha_{\rho}(s)}\left(1+O\left(\frac{1}{t}\right)\right), \\
& \frac{1}{2} \lambda^{K} \Gamma\left(1-\alpha_{\rho}(s)\right)\left(-\alpha_{\phi}^{\prime} t\right)^{\alpha_{\rho}(s)}\left(1+O\left(\frac{1}{t}\right)\right),
\end{aligned}
$$

for the three processes, respectively. Since $\alpha_{\rho}^{\prime}$ $=\alpha_{K}^{\prime}{ }^{\prime}=\alpha_{\phi}^{\prime}$ in the $t$ channel for $t \rightarrow \infty$, factorization gives

$$
\lambda^{\pi} \lambda^{K}=2\left(\lambda^{\pi K}\right)^{2} .
$$

If we further argue that the $\rho$ residues take $\mathrm{SU}(3)$ symmetric values, we have

$$
\lambda^{K}=\lambda^{\pi K}=\frac{1}{2} \lambda^{\pi} .
$$

We need only Eq. (2.3) in this and in the next section.

Next we consider the factorization constraints at the $s$-channel resonances, where the slopes are not necessarily equal. For $s \rightarrow s_{L}$ and $\alpha_{\rho}\left(s_{L}\right)=L$ we obtain

$$
\begin{aligned}
A\left(\pi^{+} \pi^{-} \rightarrow \pi^{+} \pi^{-}\right) \rightarrow & \frac{\lambda^{\pi}}{\alpha_{\rho}^{\prime}\left(s-s_{L}\right)} \frac{1}{\Gamma(L)} \\
& \left.\times \prod_{n=0}^{L-1}\left(\alpha_{\rho}(t)+n\right)\right) \\
A\left(\pi^{+} \pi^{-} \rightarrow K^{+} K^{-}\right) & \rightarrow \frac{\lambda^{\pi K}}{\alpha_{\rho}^{\prime}\left(s-s_{L}\right)} \frac{1}{\Gamma(L)} \\
& \times \prod_{n=0}^{L-1}\left(\alpha_{K} *(t)+n\right), \\
\left.A\left(K^{+} K^{-}\right) \rightarrow K^{+} K^{-}\right) \rightarrow & \frac{\lambda^{K}}{\alpha_{\rho}^{\prime}\left(s-s_{L}\right)} \frac{1}{\Gamma(L)} \\
& \times \prod_{n=0}^{L-1}\left(\alpha_{\phi}(t)+n\right) .
\end{aligned}
$$

In Eqs. (2.5a) and (2.5b), the even- and odd-spin parents and their daughters contribute to the $I=0$ and $I=1$ amplitudes, respectively. Therefore at each integer $l(0 \leqslant l \leqslant L)$, there is only one particle contributing. In Eq. (2.5c), there are two particles contributing to each value of $l$, the one with $I=0$ and the other with $I=1$. This gives rise to a factor $\frac{1}{2}$ to the resonance residues in the reaction $K^{+} K^{-}-K^{+} K^{-}$. Writing Eqs. (2.5) in terms of states with definite angular momenta, we have

$$
A\left(\pi^{+} \pi^{-} \rightarrow \pi^{+} \pi^{-}\right) \rightarrow \frac{\lambda^{\pi}}{\alpha_{\rho}^{\prime}\left(s-s_{L}\right)} \frac{1}{\Gamma(L)}\left[\left(2 \alpha_{\rho}^{\prime} k_{\pi^{2}}\right)^{L} \frac{2^{L}[\Gamma(L+1)]^{2}}{\Gamma(2 L+1)} P_{L}\left(z_{s}\right)+a_{\pi} \frac{2^{L-1}[\Gamma(L)]^{2}}{\Gamma(2 L)}\left(2 \alpha_{\rho}^{\prime} k_{\pi}{ }^{2}\right)^{L-1} P_{L-1}\left(z_{s}\right)+\cdots\right]
$$


To obtain the expressions for $A\left(\pi^{+} \pi^{-} \rightarrow K^{+} K^{-}\right)$and $A\left(K^{+} K^{-}-K^{+} K^{-}\right)$, we make the substitution

$\left(\lambda^{\pi}, \alpha_{\rho}^{\prime} k_{\pi}^{2}, a_{\pi}\right) \rightarrow\left(\lambda^{\pi K}, \alpha_{K}^{\prime} * k_{\pi} k_{K}, a_{\pi K}\right)$ and

$\left(\lambda^{K}, \alpha_{\phi}^{\prime} k_{K}^{2}, a_{K}\right)$. The quantities $k_{\pi}$ and $k_{K}$ are the

c. m. momenta of the $\pi \pi$ and $K \bar{K}$ systems, $z_{s}$ is the cosine of the c.m. scattering angle, and

$$
\begin{aligned}
a_{\pi}=L & {\left[-\frac{1}{2}-\frac{1}{2} \alpha_{\rho}(0)+2 \alpha_{\rho}\left(m_{\pi}^{2}\right)\right], } \\
a_{\pi K}=L & {\left[-\frac{1}{2}-\frac{1}{2} \alpha_{\rho}(0)+\alpha_{\rho}\left(m_{\pi}^{2}\right)+\alpha_{K^{*}}\left(m_{K}^{2}\right)\right.} \\
& \left.-\frac{1}{2}\left(\alpha_{K^{*}}^{\prime}-\alpha_{\rho}^{\prime}\right)\left(s_{L}-2 m_{\pi}^{2}\right)\right], \\
a_{K}=L & {\left[-\frac{1}{2}-\frac{1}{2} \alpha_{\rho}(0)+\alpha_{\rho}\left(m_{K}^{2}\right)+\alpha_{\phi}\left(m_{K}^{2}\right)\right.} \\
& \left.-\frac{1}{2}\left(\alpha_{\phi}^{\prime}-\alpha_{\rho}^{\prime}\right)\left(s_{L}-2 m_{K}^{2}\right)\right] .
\end{aligned}
$$

Factorization at the parent level together with Eq. (2.3) gives ${ }^{12}$

$$
\alpha_{\rho}^{\prime} \alpha_{\phi}^{\prime}=\alpha_{K^{*}}{ }^{2}
$$

Factorization of the first daughter can be achieved in the case of the degenerate slopes if the following conditions are satisfied:

$$
m_{\rho}^{2}-m_{\pi}^{2}=m_{K^{*}}{ }^{2}-m_{K}^{2}=m_{\phi}^{2}-2 m_{K}^{2}+m_{\pi}^{2},
$$

which imply the nonet mass formula $m_{\rho}{ }^{2}+m_{\phi}{ }^{2}$ $=2 m_{K^{*}}{ }^{2}$. They can also be rewritten in terms of the trajectory parameters,

$$
\alpha_{\rho}\left(m_{\pi}^{2}\right)=\alpha_{K^{*}}\left(m_{K}^{2}\right)=\alpha_{\phi}\left(2 m_{K}^{2}-m_{\pi}^{2}\right)
$$

or

$$
\alpha_{\rho}(0)+\alpha_{\phi}(0)=2 \alpha_{K^{*}}(0)
$$

or

$$
\alpha_{\rho}\left(m_{K}^{2}\right)+\alpha_{\phi}\left(m_{K}^{2}\right)=2 \alpha_{K^{*}}\left(m_{K}^{2}\right) .
$$

The last two expressions are equivalent forms of equal-spacing rules. Equation (2.10) gives the mass-quantization rules ${ }^{6}$ if it is set equal to $\frac{1}{2}$. It is clear from Eq. (2.7) that factorization of the first or subsequent younger daughters is impossible if the slopes are different. ${ }^{13}$ The condition analogous to Eq. (2.9) for the $\pi \pi \rightarrow \pi \pi, \pi \pi \rightarrow D \bar{D}$, and $D \bar{D} \rightarrow D \bar{D}$ scatterings leads to $m_{\rho}{ }^{2}-m_{\pi}{ }^{2}=m_{D *}{ }^{2}$ $-m_{D}{ }^{2}=m_{J}^{2}-2 m_{D}{ }^{2}+m_{\pi}^{2}$. The last identity is satisfied rather poorly, indicating that factorization of the first daughters cannot be maintained in general even if the slopes are degenerate, when the charmed-meson channels are included in the consideration.

\section{TRAJECTORIES FOR NONDEGENERATE SLOPES}

Our strategy in this section is to consider the two relations derived in the preceding section, the factorization of the trajectory slopes and the equalspacing rules, as basic properties of the leading meson trajectories. These two relations are, of course, not on equal footing. Factorization rela- tions [see- Eq. (3.1)] can be derived from an asymptotic symmetry such as Eq. (2.4) and the factorization of parent particles irrespective of the degeneracy or the lack of degeneracy of the slopes. The equal-spacing rule, extracted from the case of degenerate slopes, is, however, an assumption. As will be seen in the next section, the good predictions which follow indicate the validity of this assumption. From the above argument, we can write the factorization conditions as

$$
\begin{aligned}
& \alpha_{\rho}^{\prime} \alpha_{\phi}^{\prime}=\alpha_{K^{*}}^{\prime}, \\
& \alpha_{\rho}^{\prime} \alpha_{J}^{\prime}=\alpha_{D}^{\prime *^{2},} \\
& \alpha_{\phi}^{\prime} \alpha_{J}^{\prime}=\alpha_{F *}^{\prime}{ }^{2},
\end{aligned}
$$

and the equal-spacing rules as

$$
\begin{aligned}
& \alpha_{\rho}(0)+\alpha_{\phi}(0)=2 \alpha_{K^{*}}(0), \\
& \alpha_{\rho}(0)+\alpha_{J}(0)=2 \alpha_{D^{*}}(0), \\
& \alpha_{\phi}(0)+\alpha_{J}(0)=2 \alpha_{F}(0),
\end{aligned}
$$

which we generalized from Eq. (2.11a). The generalization of Eq. (2.11b) is

$$
\begin{aligned}
& \alpha_{\rho}\left(m_{K}^{2}\right)+\alpha_{\phi}\left(m_{K}^{2}\right)=2 \alpha_{K^{*}}\left(m_{K}^{2}\right), \\
& \alpha_{\rho}\left(m_{D}^{2}\right)+\alpha_{J}\left(m_{D}^{2}\right)=2 \alpha_{D^{*}}\left(m_{D}^{2}\right), \\
& \alpha_{\phi}\left(m_{F}^{2}\right)+\alpha_{J}\left(m_{F}^{2}\right)=2 \alpha_{F}\left(m_{F}^{2}\right) .
\end{aligned}
$$

Equations (3.2) and (3.3) are alternative equalspacing rules. They cannot hold simultaneously, otherwise a common slope will result for all the trajectories. We do not propose to use relations such as (2.10), which gives directly ratios of slopes. They are inconsistent with Eq. (3.1), unless nonet types of mass formulas hold. The second and third lines of Eq. (3.1) are derived from the following sets of amplitudes, following a similar derivation as outlined in Sec. II:

$$
\begin{aligned}
A\left(\pi^{+} \pi^{-}-\bar{D}^{0} D^{\circ}\right) & =\lambda^{\pi D} V_{\rho D^{*}}(s, t), \\
A\left(D^{0} \bar{D}^{0}-D^{\circ} \bar{D}^{\circ}\right)=\frac{1}{2} \lambda^{D}[ & V_{\rho J}(s, t)+V_{J \rho}(s, t) \\
& \left.+V_{\omega J}(s, t)+V_{J \omega}(s, t)\right],
\end{aligned}
$$

together with Eq. (2.1a) and $\lambda^{\pi} \lambda^{D}=2\left(\lambda^{\pi D}\right)^{2}$, and

$$
A\left(K^{+} K^{-} \rightarrow \bar{D}^{0} D^{0}\right)=\frac{1}{2} \lambda^{K D}\left[V_{\rho F^{*}}(s, t)+V_{\omega F^{*}}(s, t)\right],
$$

together with Eqs. (2.1c) and (3.4b) and $\lambda^{K} \lambda^{D}$ $=\left(\lambda^{K D}\right)^{2}$. If we further assume asymptotic symmetry for the $\rho$ residue, we have $\lambda^{D}=\lambda^{\pi D}=\frac{1}{2} \lambda^{\pi}$ and $\lambda^{K}=\lambda^{D}=\lambda^{K D}$.

Equations (3.1) and (3.2) give $\mathrm{e}^{14}$

$$
\begin{aligned}
& \alpha_{K}^{\prime}=\alpha_{\rho}^{\prime} \frac{m_{K^{*}}^{2}}{m_{\phi}^{2}}\left[1+\left(1-m_{\rho}^{2} m_{\phi}^{2} / m_{K} *^{4}\right)^{1 / 2}\right], \\
& \alpha_{D^{*}}^{\prime}=\alpha_{\rho}^{\prime} \frac{m_{D^{*}}{ }^{2}}{m_{J}^{2}}\left[1+\left(1-m_{\rho}{ }^{2} m_{J}{ }^{2} / m_{D} *^{2}\right)^{1 / 2}\right] .
\end{aligned}
$$


Relations similar to (3.6) can be derived from Eqs. (3.1) and (3.3) with $m_{\rho}{ }^{2}, m_{K^{*}}{ }^{2}$, and $m_{\phi}{ }^{2}$ replaced by $m_{\rho}{ }^{2}-m_{K}{ }^{2}, m_{K}{ }^{2}-m_{K}{ }^{2}$, and $m_{\phi}{ }^{2}-m_{K}{ }^{2}$ in $\alpha_{K^{*}}^{\prime}$, and $m_{\rho}{ }^{2}, m_{D^{*}}{ }^{2}$, and $m_{J}{ }^{2}$ by $m_{\rho}{ }^{2}-m_{D}{ }^{2}, m_{D}{ }^{2}$ $-m_{D}{ }^{2}$, and $m_{J}{ }^{2}-m_{D}{ }^{2}$ in $\alpha_{D^{*}}^{\prime}$. Note that when the nonet mass relations, i.e., $m_{\rho}{ }^{2}+m_{\phi}{ }^{2}=2 m_{K^{*}}{ }^{2}$ and $m_{\rho}^{2}+m_{J}{ }^{2}=2 m_{D} *^{2}$, become exact, Eq. (3.6) leads to degenerate slopes, $\alpha_{\rho}^{\prime}=\alpha_{K^{*}}^{\prime}=\alpha_{D^{*}}^{\prime}$, etc. Equations (3.1) and (3.3) also give a universal slope if mass relations such as $m_{\rho}{ }^{2}-m_{\pi}{ }^{2}=m_{K}{ }^{2}-m_{K}{ }^{2}=m_{D}{ }^{2}-m_{D}{ }^{2}$ hold. ${ }^{15}$ The nonet mass relations are assumed to hold with the presence of first-order symmetrybreaking effects, suggesting that the nondegenerate slopes are due to symmetry-breaking effects higher than the first order. ${ }^{16}$ We shall come back to this point again in the next section. Taking $m_{\rho}$ $=0.768, m_{K^{*}}=0.894, m_{\phi}=1.02, m_{D^{*}}=2.01$, and $m_{J}$ $=3.098$, all in units of $\mathrm{GeV},{ }^{17}$ we obtain

$$
\begin{aligned}
& \alpha_{K^{*}}^{\prime} / \alpha_{\rho}^{\prime}=0.924, \quad \alpha_{\phi}^{\prime} / \alpha_{\rho}^{\prime}=0.854, \\
& \alpha_{D^{*}}^{\prime} / \alpha_{\rho}^{\prime \prime}=0.761, \quad \alpha_{J}^{\prime} / \alpha_{\rho}^{\prime}=0.579, \\
& \alpha_{F}^{\prime} / \alpha_{\rho}^{\prime}=0.703 .
\end{aligned}
$$

Equations (3.1) and (3.3) give ratios of slopes about $3 \%$ higher than the above. This demonstrates the stability of the equal-spacing rules.

\section{SYMMETRY BREAKING AND DECAY WIDTH}

Consider the elastic scattering of two pseudoscalar mesons, $a+b \rightarrow a+b$, which is described by the following amplitude:

$A=\lambda V_{A B}(s, t)+$ possible terms with different $s$-channel poles.

For $s=m_{L}^{2}$ and $\alpha\left(m_{L}^{2}\right)=L$,

$$
A=\frac{\lambda}{\alpha_{A}^{\prime}} \frac{1}{\Gamma(L)} \frac{2^{L}[\Gamma(L+1)]^{2}}{\Gamma(2 L+1)}\left(2 \alpha_{B}^{\prime} k^{2}\right)^{L} \frac{P_{L}(\cos \theta)}{s-m_{L}^{2}}+\cdots
$$

Only particles on the leading trajectory are considered. The decay width of this particle $L$ to $a+b$ is given in the narrow-resonance limit as

$$
\begin{aligned}
\Gamma_{L \rightarrow a+b} & =\xi_{L} \frac{k}{16 \pi m_{L}^{2}} \lim _{s \rightarrow m_{L}{ }^{2}}\left(s-m_{L}^{2}\right) \int_{-1}^{1} d(\cos \theta) P_{L}(\cos \theta) A \\
& =\frac{\lambda}{16 \pi} \xi_{L}\left(\alpha_{\rho}^{\prime}\right)^{L-1}\left[\left(\frac{\alpha_{\rho}^{\prime}}{\alpha_{A}^{\prime}}\right)\left(\frac{\alpha_{B}^{\prime}}{\alpha_{\rho}^{\prime}}\right)^{L}\right] \frac{2^{L+1} L}{(2 L+1) ! !} \frac{k^{2 L+1}}{m_{L}{ }^{2}},
\end{aligned}
$$

where $\xi_{L}$ is the isospin factor. We normalize the width in terms of $\boldsymbol{\alpha}_{\rho}^{\prime}$. The factor in the square brackets,

$$
\left(\frac{\alpha_{\rho}^{\prime}}{\alpha_{A}^{\prime}}\right)\left(\frac{\alpha_{B}^{\prime}}{\alpha_{\rho}^{\prime}}\right)^{L},
$$

gives rise to a symmetry breaking which can also be seen from the coupling constants. For the vector- and tensor-meson couplings one has

$$
\begin{aligned}
& g_{V a b}^{2}=\frac{1}{2} \lambda \xi_{1}\left(\frac{\alpha_{\rho}^{\prime}}{\alpha_{A}^{\prime}}\right)\left(\frac{\alpha_{B}^{\prime}}{\alpha_{\rho}^{\prime}}\right), \\
& g_{T a b}^{2}=\frac{1}{4} \lambda \xi_{2}\left(\frac{\alpha_{\rho}^{\prime}}{\alpha_{A}^{\prime}}\right)\left(\frac{\alpha_{B}^{\prime}}{\alpha_{\rho}^{\prime}}\right)^{2} \alpha_{\rho}^{\prime} .
\end{aligned}
$$

Equation (4.3) predicts a large symmetry-breaking effect for high-spin resonances on the $\rho-f-\omega-$ $A_{2}$ trajectory decaying into $K \bar{K}$ or $D \bar{D}$. A ready interpretation of this symmetry-breaking effect can be given in terms of quark diagrams. Suppose that the decaying particle $L$ is made of quarks $q_{1} \bar{q}_{2}$, particle $a, q_{1} \bar{q}_{3}$ and particle $b, q_{3} \bar{q}_{2}$. We shall call $q_{1} \bar{q}_{2}$ the initial quarks and $q_{3} \bar{q}_{3}$ the paircreated quarks. Then $\alpha_{A}^{\prime}$ is the slope of the trajectory made of the initial quarks, and $\alpha_{B}^{\prime}$ is that made of the pair-created quarks. (See Fig. 1.) Thus, $\alpha_{A}^{\prime}$ is the slope of the trajectory on which the decaying particle lies and $\alpha_{B}^{\prime}$ can only be $\alpha_{\rho}^{\prime}$, $\boldsymbol{\alpha}_{\phi}^{\prime}$, or $\boldsymbol{\alpha}_{J}^{\prime}$. Therefore the first factor of Eq. (4.3), $\alpha_{\rho}^{\prime} / \alpha_{A}^{\prime}$, gives rise, in general, to an enhancement and the second factor, $\left(\alpha_{B}^{\prime} / \alpha_{\rho}^{\prime}\right)^{L}$, to a suppression. The latter can be understood intuitively as follows. The creation of a pair of heavy quarks from the vacuum is less probable than that of a pair of light quarks. This type of argument has been used in the literature in the discussion of couplings of $D \bar{D}$ (and $F \bar{F}$ ) to the ordinary vector mesons and to $J / \psi$ and $\psi^{\prime}$. The presence of the first factor $1 / \alpha_{A}^{\prime}$ is expected from the general formulas of the width of a resonance on a Regge trajectory. ${ }^{18}$

In order to determine all the trajectories and the widths given in Eq. (4.2), we use $\alpha_{\rho}^{\prime}$ and the 


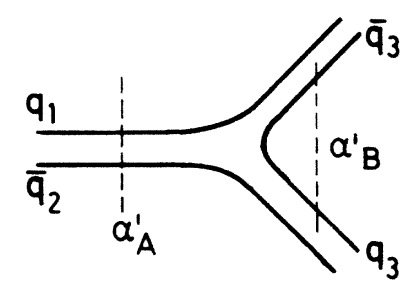

FIG. 1. Quark-diagram interpretation of the symmetrybreaking effect of Eq. (4.3).

decay width $\Gamma_{\rho \rightarrow 2 \pi}$ as inputs. The latter involves the least error of all the vector-meson decay widths and the former has been repeatedly determined in the spacelike region. We shall use $\alpha_{\rho}^{\prime}$ $\simeq 0.88 \mathrm{GeV}^{-2}$, which comes from the following information: (a) The $\rho-f-\omega-A_{2}$ trajectory goes through $h$ (2.04) (Ref. 19) for $\alpha_{\rho}\left(m_{h}{ }^{2}\right)=4$. (b) A recent fit of the $\pi N$ charge-exchange reaction ${ }^{20}$ gives $\alpha_{\rho}(0)=0.48$, which leads to $\alpha_{\rho}^{\prime}=0.88 \mathrm{GeV}^{-2}$ when it is extrapolated to the timelike region and required to pass through the $\rho$.

Let us restrict ourselves in the following discussion to the equal-spacing rule, Eq. (3.2). In Table I, we list the resulting slope parameters and the predicted mass values together with the experimental masses if they exist. Input masses are underlined. The values of the slopes are close to those used by Igi. ${ }^{4}$ We note the following: (a) The resonance at $J=3$ on the $K^{*}-K^{* *}$ trajectory with the predicted mass $1.805 \mathrm{GeV}$ can be identified with $K_{N}(1.8) .^{21}$ (b) The predicted mass 3.677 on the $\alpha_{J}$ with $\alpha_{J}=3$ agrees well with that of $\psi^{\prime}(3.684)$, since $\psi^{\prime}(3.684)$ should be identified as the second daughter of $\alpha_{J}=3$ resonance on the $\alpha_{J}$. (c) The $F^{*}$ mass is predicted to be $2.14 \mathrm{GeV}$, which is higher than the prediction of $2.06 \mathrm{GeV}$ in the charmonium model. ${ }^{22}$ We observe, however,

TABLE I. Predicted mass spectra and partial widths into two pseudoscalar mesons. (i) The underlined vector-meson masses, $\alpha_{\rho}^{\prime}=0.88 \mathrm{GeV}^{-2}$, and the $\rho \rightarrow 2 \pi$ width are input. (ii) The decay, $D^{* 0} \rightarrow D^{0} \pi$, is very close to the threshold; therefore, the calculated width is extremely sensitive to the masses of $D^{* 0}$ and $D^{0}$ used. See Ref. 22. (iii) $R_{a b} \equiv \alpha_{a}^{\prime} / \alpha_{b}^{\prime}$.

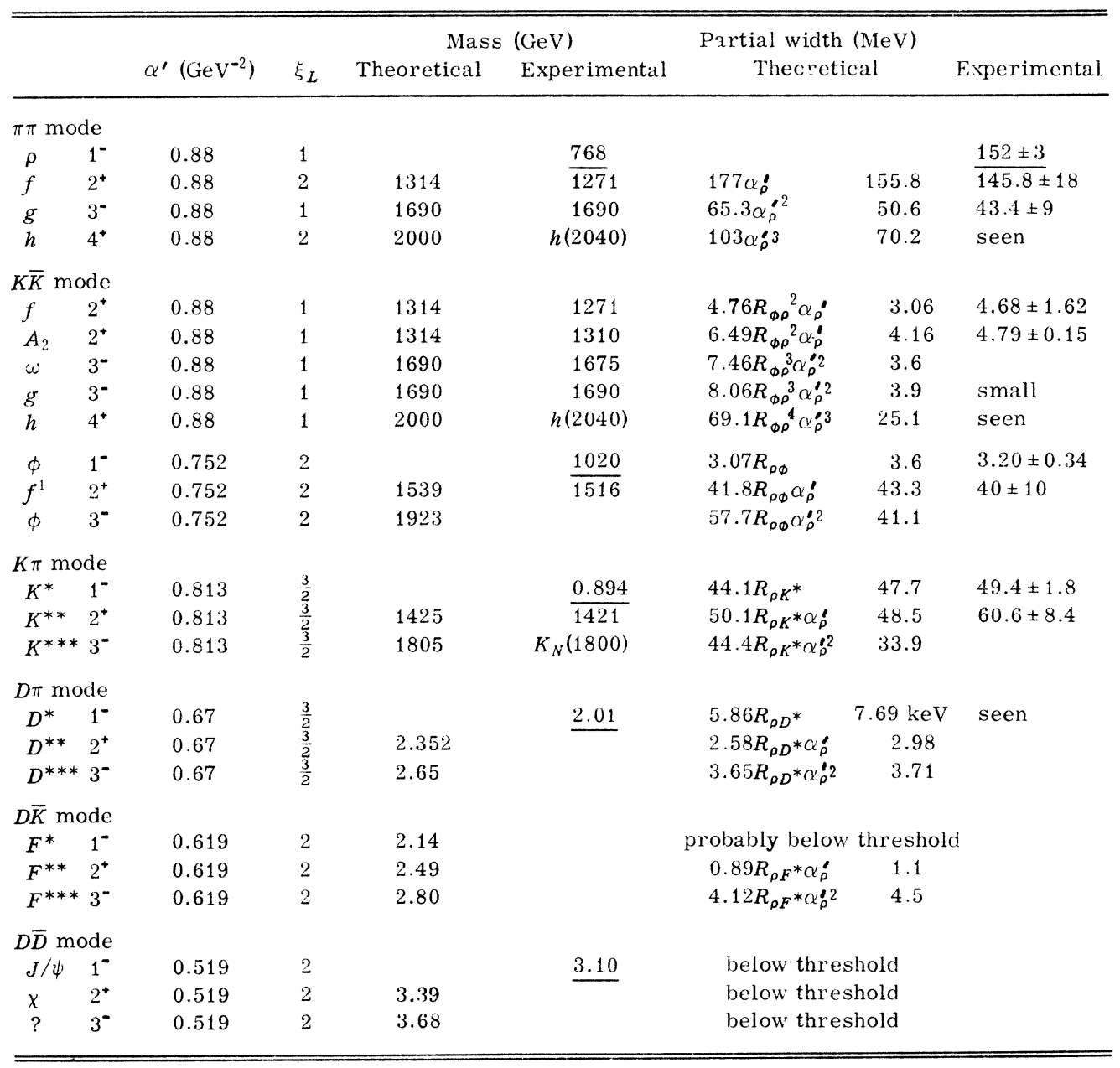


that the prediction of the charmonium model of the masses of $D, D^{*}$, and $\Lambda_{c}(2.25)$ are in general lower by 20-30 MeV compared with the experimental values. ${ }^{1}$ (d) The mass-quantization rules, which are not part of our inputs, are satisfied reasonably well for the known pseudoscalar mesons. Using the $\rho, K^{*}, D^{*}, F^{*}$, and $J / \psi$ trajectories in $\alpha_{V}\left(m_{P}{ }^{2}\right)=\frac{1}{2}$, where $m_{P}$ is the corresponding pseudoscalar mass, we obtain the first three relations from the Adler PCAC (partial conservation of axial-vector current) consistency condition but the last two cannot be derived this way. Solving these relations, we obtain $m_{\pi}=0.147, m_{K}=0.43, m_{D}$ $=1.82, m_{F}=1.94$, and $m_{\eta_{c}}=2.94$, all in units in GeV.

The various decay widths into two pseudoscalars are also given in Table I. Except for the $\rho \rightarrow 2 \pi$ width, which is used as an input to fix $\lambda^{\pi}$, all the other widths listed are predictions. Experimental values of masses are used in the calculation if available. We also give the isospin factor $\xi_{L}$ [see Eq. (4.2)] and the experimental widths. For the $D^{*}$, the width listed is for $D^{* 0}-D^{0} \pi^{0}$, which is barely above the threshold and has been observed. $^{23}$ To indicate the symmetry-breaking effects, we also list the widths in terms of the slopes. All the predicted widths suffer an error of the order of $4 \%$, mainly due to the ambiguity of the value of $\alpha_{\rho}^{\prime}$ and the assumption of exact exchange degeneracy.

To conclude this section, a remark on $D^{* 0}-D^{0} \pi^{0}$ is in order. It is very close to the threshold; therefore, the calculated width, strongly suppressed by the limited phase space, is sensitive to the mass values of $D^{* 0}$ and $D^{0}$ used. (We used the values given in Ref. 22.) Although the predicted width is of the order of $\mathrm{keV},{ }^{23}$ the predicted coupling constant for $D^{*} \bar{D}^{0} \pi$ is larger than the $\mathrm{SU}(4)$-symmetry value by the factor $\left(\alpha_{\rho}^{\prime} / \alpha_{D^{*}}^{\prime}\right)^{1 / 2}$.

\section{CONCLUSIONS}

We emphasized that relations, such as the nonet mass formula, the equal-spacing rule, and the mass quantization rule, are related to the factorization requirement at the first daughter level in the case of the universal slope. Then we proposed that the facotrization relation and equal-spacing rule, which relate the parameters of different trajectories, hold even when the trajectory slopes are different, and thereby we determined the parameters of all the leading meson trajectories in terms of the slope of the $\rho$ trajectory. The predicted decay widths of vector mesons, tensor mesons, etc. into two pseudoscalars agree well with the data whenever a comparison can be made. The nondegenerate slopes improve the prediction of the corresponding degenerate slopes. This can be seen in, e.g., $\Gamma_{K^{*}(890) \rightarrow K \pi}$, which is independent of the ab- solute value of $\alpha_{\rho}^{\prime}$. The predicted partial width agrees with the data within the experimental error, while the degenerate slopes, giving rise to exact $\mathrm{SU}(3)$ - [SU(4) - I symmetric couplings, predict a value which is three standard deviations too small in comparison with the data. Another interesting case is $D^{* 0}-D^{0} \pi^{0}$ in which the predicted width is larger than the SU(4)-symmetric value by $30 \%$. Note that the $D^{*} \bar{D} \pi$ coupling constant is dimensionless; therefore, it is not clear how to introduce a mass scale into the effective Lagrangian which can account for symmetry-breaking effects. In the present approach the scales are provided by the slope parameters whose ratios are determined by the factorization condition as well as the equalspacing rule. An accurate measurement of the $D^{* 0} \rightarrow D^{0} \pi^{0}, K^{* *}(1420) \rightarrow K \pi$, and $f \rightarrow K \bar{K}$ widths could serve as additional tests of the present scheme, in particular, as a test of the equal-spacing rules. A direct check of the equal-spacing rules can be achieved if the masses of recurrences of the various trajectories, as listed in Table I, are experimentally determined. Let us further notice that strong suppression will occur in the $K \bar{K}$ and $D \bar{D}$ mode of high-spin particles lying on the $\rho-f-\omega-A_{2}$ trajectory, owing to the factors $\left(\alpha_{K^{*}}^{\prime} / \alpha_{\rho}^{\prime}\right)^{L}$ and $\left(\alpha_{D^{*}}^{\prime} / \alpha_{\rho}^{\prime}\right)^{L}$. An experimental check of these factors is possible for the former but remote for the latter.

There are other schemes beside ours which discuss nondegenerate slopes. One of them, proposed by Close et al. ${ }^{24}$ assumes the following relation between the slope $\alpha_{\boldsymbol{V}}^{\prime}$, of a leading trajectory and the lowest vector-meson mass on it, $m_{V}: \alpha_{V}^{\prime}$ $=\frac{3}{4} m_{V}{ }^{-1}$. These slope parameters do not satisfy the factorization condition (3.1). Another scheme, proposed by Finkelstein and Tuan, ${ }^{4}$ in which $\alpha_{\rho}^{\prime}$ $\simeq \alpha_{D^{*}}^{\prime}$, etc. are assumed, leads to degenerate slopes when the factorization of the resonance residues is assumed.

As a concluding remark, beyond the present phenomenological level, a more precise formulation of the dual resonance model with nondegenerate slopes is necessary. In this formulation, it is necessary to define, among other things, the energy range outside of which the slopes become universal. This range was left unspecified in the present phenomenological treatment.

\section{ACKNOWLEDGMENT}

We would like to thank T. Walsh, M. Krammer, J. Körner, and T. C. Yang for reading the manuscript and helpful suggestions. M.K. thanks the Alexander von Humboldt Foundation for financial support. B.L.Y. takes the pleasure of thanking Professor H. Joos and Dr. T. Walsh for their kind hospitality at DESY and for financial support. 
*Work supported by Alexander von Humboldt Foundation. $\dagger$ On sabbatical leave at DESY, 1976-1977. Work supported in part by ERDA under Contract No. W-7405Eng-82.

$\$$ Permanent address.

${ }^{1}$ G. Goldhaber et al., Phys. Rev. Lett. 37, 255 (1976); DASP Collaboration, W. Braunschweig et al., Phys. Lett. 63B, 471 (1976); I. Perzzi et al ., Phys. Rev. Lett. 37, 569 (1976); PLUTO Collaboration, J. Burmester et al ., Phys. Lett. 64B, 369 (1976). For a summary of the more recent state of affairs, see B. H. Wiik, Invited talk at the 18th International Conference on High Energy Physics, Tbilisi, U.S.S.R. [DESY Report No. DESY 76/52, 1976 (unpublished)]; H. Meyer, Invited talk given at the 1977 Coral Gables Conference, Report No. DESY 77/19 (unpublished).

${ }^{2}$ For a review see, for example, M. Jacob, in Proceedings of the Schladming Winter School, 1969, edited by P. Urban (Springer, New York, 1969), or J. J. Peterson, Phys. Rep. 2C, 157 ( 971)

${ }^{3}$ D. W. McKay and Bing-Lin Young, Phys. Rev. D 15 , 1282 (1977); J. Finkelstein and S. S. Pinsky, ibid. $\underline{15}$, 360 (1977).

${ }^{4}$ K. Igi, Phys. Rev. D $\underline{15}, 865$ (1977); Phys. Lett. 66B, 276 (1977); J. Finkelstein and S. F. Tuan, Phys. Rev. D 15,902 (1977).

${ }^{5} \mathrm{M}$. Ademollo, G. Veneziano, and S. Weinberg, Phys. Rev. Lett. 22, 83 (1969).

${ }^{6} \mathrm{M}$. Krammer (private communication). A candidate for the $\eta_{c}$ has been seen by the DESY DASP group [called $\mathrm{X}(2.8)]$; W. Braunschweig et al., Phys. Lett. 67B, 243 (1977); 67B, 249 (1977).

${ }^{7}$ S. Mandelstam, Phys. Rev. Lett. 21, 1724 (1968).

${ }^{8} \mathrm{~K}$. Igi, in Ref. 4.

${ }^{9} \mathrm{~K}$. Kawarabayashi, S. Kitakado, and H. Yabuki, Phys. Lett. 28B, 432 (1969).

${ }^{10}$ F. Takagi, Prog. Theor. Phys. 41, 1555 (1969);

P. Freund, Lett. Nuovo Cimento 1 , 928 (1969).

${ }^{11}$ The actual values of slope parameters of the $D^{*}$ and $J / \psi$ trajectories are crucial in a certain model calculation of charmed-meson and -baryon production; see for example, C. Avilez, T. Kobayashi, and J. G. Körner, Phys. Lett. 66B, 149 (1977); Report No. DESY 66B, 149 (1977); Phys. Rev. (to be published).

${ }^{12}$ This relation has been obtained by J. Pasupathy [Phys. Rev. Lett. 37, 1336 (1976)] and by Igi (Ref. 6) by means of factorization of the Regge expansion in the $t$ vari- able of, say, Eq. (2.1) in the medium-energy region. Such a procedure, which requires the validity of asymptotic expansion of $\Gamma$ functions, leads to an exponential increase (Ref. 7) of the $\pi K \rightarrow \pi K$ amplitude at fixed angle $\theta$, for $\cos \theta<1-2 \alpha_{K}^{*} / \alpha_{\rho}^{\prime}$. We do not regard this exponential increase as a reasonable behavior to occur in the medium energy region, although it disappears eventually as $t \rightarrow \infty$. Our derivation of Eq. (2.8) is carried out in the resonance region and does not suffer from this difficulty.

${ }^{13}$ The second and younger daughters are not factorizable even in the case of degenerate slope. See Freund, Ref. 10.

${ }^{14}$ We discard another solution, e.g.

$$
\alpha_{K^{*}}^{\prime}=\alpha_{\rho}^{\prime} \frac{m_{K^{*}}{ }^{2}}{m_{\phi}^{2}}\left[1-\left(1-m_{\rho}^{2} m_{\phi}^{2} / m_{K^{*}}{ }^{4}\right)^{1 / 2}\right]
$$

etc., on the ground that it gives $\alpha_{K^{*}}{ }^{*}=0$ when the nonet mass relation $m_{\rho}^{2}+m_{\phi}^{2}=2 m_{K^{*}}{ }^{2}$ is exact.

${ }^{15}$ These relations are derived from the quantization rules with universal slope; see McKay and Young, Ref. 3.

${ }^{16} \mathrm{Higher}$-order symmetry-breaking effects in the GellMann-Okubo mass formula have been considered by S. Okubo, Phys. Lett. 4 , 74 (1963), and M. A. Rashid and I. I. Yamanaka, Phys. Rev. 131, 2797 (1963).

${ }^{17}$ The values of $m_{\rho}$ and $m_{K} *$ used here are the average of the charged and neutral masses given in Particle Data Group, Rev. Mod. Phys. 48, S1 (1976).

${ }^{18}$ See, for example, S. C. Frautschi, Regge Poles and S-Matrix Theory (Benjamin, New York, 1963).

${ }^{19}$ W. D. Apel et al ., Phys. Lett. 57B, 398 (1975); W. Blum et al ., ibid. 57B, 430 (1975).

${ }^{20} \mathrm{~A}$. V. Barnes et al ., Phys. Rev. Lett. 37, 72 (1976).

${ }^{21}$ A. Firestone et al ., Phys. Lett. 36B, 513 (1971); G. W. Brandenburg et al ., ibid. $\underline{60 \mathrm{~B}}, 478$ (1976). ${ }^{22}$ A. De Rajula et al., Phys. Rev. D $\underline{12}, 147$ (1975).

${ }^{23} \mathrm{G}$. Goldhaber, Report No. LBL-5534 (unpublished). In the calculation of $\Gamma_{D^{* 0} \rightarrow D^{0} \pi^{0}}$ we used the mass values quoted in this article: $m_{D^{0}}=1.8565, m_{D^{* 0}}=2.0067$. In all the other width calculations involving $D$ and $\pi$ we used $m_{D}=1.87$ and $m_{\pi}=0.137$. This article also gives $\Gamma\left(D^{* 0} \rightarrow D^{0} \gamma\right) / \Gamma\left(D^{* 0} \pi^{0}\right)=0.54 \sim 0.82$, which indicated that the experimental width of $D *_{0} \rightarrow D^{0} \pi$ is probably of the order of keV.

${ }^{24}$ F. E. Close, D. M. Scott, and D. Sivers, Phys. Lett. 62B, 213 (1976). 